\title{
Macroporous Composite Cryogels with Embedded Polystyrene Divinylbenzene Microparticles for the Adsorption of Toxic Metabolites from Blood
}

\author{
Tanja Eichhorn, ${ }^{1}$ Alexander E. Ivanov, ${ }^{2}$ Maria B. Dainiak, ${ }^{2}$ André Leistner, ${ }^{3}$ \\ Ingrid Linsberger, ${ }^{1}$ Hans Jungvid, ${ }^{2}$ Sergey V. Mikhalovsky, ${ }^{4,5}$ and Viktoria Weber ${ }^{1}$ \\ ${ }^{1}$ Center for Biomedical Technology, Danube University Krems, Dr.-Karl-Dorrek-Strasse 30, 3500 Krems, Austria \\ ${ }^{2}$ Protista Biotechnology AB, Kvarngatan 2, 26734 Bjuv, Sweden \\ ${ }^{3}$ Polymerics GmbH, Landsberger Allee 378, 12681 Berlin, Germany \\ ${ }^{4}$ School of Pharmacy and Biomolecular Sciences, University of Brighton, Brighton BN2 4GJ, UK \\ ${ }^{5}$ Nazarbayev University, 53 Kabanbay Batyr Avenue, Astana 010000, Kazakhstan
}

Correspondence should be addressed to Viktoria Weber; viktoria.weber@donau-uni.ac.at

Received 2 February 2013; Accepted 25 March 2013

Academic Editor: Mehmet Senel

Copyright (C) 2013 Tanja Eichhorn et al. This is an open access article distributed under the Creative Commons Attribution License, which permits unrestricted use, distribution, and reproduction in any medium, provided the original work is properly cited.

\begin{abstract}
Composite monolithic adsorbents were prepared by the incorporation of neutral polystyrene divinylbenzene (PS-DVB) microparticles into macroporous polymer structures produced by cryogelation of agarose or poly(vinyl alcohol). The composite materials exhibited excellent flow-through properties. Scanning electron microscopy of the composite cryogels revealed that the microparticles were covered by thin films of poly(vinyl alcohol) or agarose and thus were withheld in the monolith structure. Plain PS-DVB microparticles showed efficient adsorption of albumin-bound toxins related to liver failure (bilirubin and cholic acid) and of cytokines (tumor necrosis factor-alpha and interleukin-6). The rates of adsorption and the amount of adsorbed factors were lower for the embedded microparticles as compared to the parent PS-DVB microparticles, indicating the importance of the accessibility of the adsorbent pores. Still, the macroporous composite materials showed efficient adsorption of albuminbound toxins related to liver failure as well as efficient binding of cytokines, combined with good blood compatibility. Thus, the incorporation of microparticles into macroporous polymer structures may provide an option for the development of adsorption modules for extracorporeal blood purification.
\end{abstract}

\section{Introduction}

Monolithic cryogels are produced in a semi-frozen aqueous state by radical polymerization of water-soluble monomers or by cross-linking of water-soluble polymers [1-3]. Ice crystals grow during freezing and finally occupy about $90 \%$ of the volume, while the chemical reactions take place in a highly concentrated unfrozen liquid microphase. After defrosting, voids appear as a result of ice melting and form an interconnected macroporous polymer structure (MPPS) with pore diameters up to 200 to $300 \mu \mathrm{m}$, whereas the pore walls are represented by thin and dense sheets of the cross-linked polymer. Cryogel monoliths are typically elastic, spongy, and highly permeable for aqueous media. Various applications of cryogels in biotechnology and biomedical sciences have been reported [1,4-7]. In particular, cryogels and hydrogels based on poly(vinyl alcohol) and agarose are low-cost, nontoxic, and biocompatible polymers and have been employed for the production of biomaterials and cell culture scaffolds [813]. Both PVA- and agarose-based cryogels are characterized by supermacroporous morphology, but differences in their overall properties exist. Cryogels made of agarose are rigid and brittle, whereas PVA-based cryogels possess a high degree of elasticity and toughness. A series of reactive and functional cryogels was developed [3-5], including cryogels capable of removing toxins from protein solutions [14, 15]. 
Here, we report on the application of PVA- and agarose-based MPPS to remove toxic metabolites related to liver failure and cytokines from blood plasma.

Liver failure is associated with a diminished detoxification capability of the liver, resulting in the accumulation of hydrophobic toxic metabolites in the blood. Metabolites such as bile acids, bilirubin, tryptophan, and phenolic compounds are albumin-bound, and thus, they cannot be removed by diffusive or convective extracorporeal blood purification systems, but require adsorptive techniques for depletion [16, 17]. Extracorporeal liver support systems apply columns containing polystyrene divinylbenzene (PS-DVB) copolymers to remove toxic metabolites via hydrophobic interactions [18-20]. In particular, neutral PS-DVB copolymers of adequate porosity ( $8-9 \mathrm{~nm}$ pore diameter) are efficient adsorbents for albumin-bound toxic metabolites [21]. In addition to extracorporeal blood purification, the depletion of toxic metabolites from human plasma is also relevant for research purposes. This was demonstrated by in vitro studies investigating the effect of the removal of toxic metabolites related to liver failure from plasma on cultured hepatocytes [22]. These and similar research applications require simple adsorbent devices with low resistance to the flow of plasma in combination with the fast adsorption of toxins. The latter can be achieved by using small adsorbent particles, providing a large outer surface area. However, the dense packing of small particles supported by filters of adequate porosity creates significant flow resistance. Therefore, a promising approach to the design of effective adsorbents may be the incorporation of PS-DVB copolymer microparticles into highly permeable monolithic MPPS, which are well compatible with common low-pressure chromatography columns or syringes.

The aim of this study was to produce monolithic composite cryogels based on poly (vinyl alcohol) and agarose with embedded PS-DVB microparticles and to characterize them with respect to flow through, adsorption, mechanical stability and biocompatibility. Since the pore size distribution and the accessibility of the pores is critical for the adsorption characteristics of PS-DVB microparticles, we assessed the influence of the embedding procedure on the accessibility of the adsorbent pores by comparing plain PS-DVB particles and PS-DVB particles embedded into monolithic MPPS with respect to their adsorption characteristics for cytokines and hydrophobic metabolites related to liver failure.

\section{Materials and Methods}

2.1. Materials. Fresh frozen human plasma was obtained from a local plasma donation center (Retz, Austria). To remove cryoprecipitates, the plasma was centrifuged at $3,600 \mathrm{~g}$ for $10 \mathrm{~min}$ at room temperature after thawing. Alternatively, blood was drawn from healthy volunteers into tubes containing trisodium citrate (Greiner Bio-One, Kremsmünster, Austria) after informed consent of the donors. To obtain plasma, the blood was centrifuged for $10 \mathrm{~min}$ at 3,600 g without brake. Bilirubin, cholic acid, phenol, tryptophan, glutaraldehyde, and sodium cyanoborohydride were purchased from Sigma-Aldrich (Steinheim, Germany).
All other chemicals were obtained from Merck (Darmstadt, Germany). Poly(vinyl alcohol) (PVA, Mowiol 18-88) was purchased from Clariant (Frankfurt am Main, Germany) and agarose was obtained from Fluka Chemie (Buchs, Switzerland).

2.2. Polystyrene Divinylbenzene Copolymers. Polystyrene divinylbenzene copolymer microparticles were provided by Polymerics GmbH, Berlin, Germany [23]. The average pore size of the microparticles was $7.7 \mathrm{~nm}$ and the BET surface was $680 \mathrm{~m}^{2}$ per $\mathrm{g}$. The total pore volume of the particles was $1.37 \mathrm{~mL} / \mathrm{g}$, with a micropore volume (pores below $2 \mathrm{~nm}$ ) of $0.27 \mathrm{~mL} / \mathrm{g}$, a mesopore volume (pores between 2 and $50 \mathrm{~nm}$ ) of $0.88 \mathrm{~mL} / \mathrm{g}$, and a macropore volume (pores above $50 \mathrm{~nm}$ ) of $0.22 \mathrm{~mL} / \mathrm{g}$. Prior to use, the adsorbent particles were washed in ethanol, distilled water, and $0.9 \%(\mathrm{w} / \mathrm{v})$ saline solution, followed by centrifugation at $3,500 \mathrm{~g}$ for $5 \mathrm{~min}$ after each washing step. The size distribution was determined using a Mastersizer 2000 (Malvern Instruments Ltd., Malvern, UK). The average particle size was $85.5 \mu \mathrm{m}$.

2.3. Preparation of Cryogels. Monolithic cryogels used in this study are available from Protista (Bjuv, Sweden) under the trademark of MPPS (macroporous polymeric structures). Synthesis of the monolithic MPPS cryogels was carried out using a liquid bath cryostat (Proline RP 1840, LaudaKoenigshofen, Germany) and an air bath cryostat (Arctest Oy, Espoo, Finland). All gels were prepared in $2 \mathrm{~mL}$ syringes in order to obtain monoliths with a diameter of $9 \mathrm{~mm}$ and a length of $20 \mathrm{~mm}$.

For the preparation of PVA cryogels, 4.5\% (w/v) PVA solution in $0.1 \mathrm{M} \mathrm{HCl}$ was put into an ice bath and kept at $4^{\circ} \mathrm{C}$ for $30 \mathrm{~min}$. Glutaraldehyde $(50 \%$, w/v) was added to the cooled solution at a final concentration of $1 \%(\mathrm{w} / \mathrm{v})$ and the mixture was precooled for $60 \mathrm{~min}$ in the liquid bath cryostat at $-12^{\circ} \mathrm{C}$ and subsequently transferred to the air bath cryostat where the samples were kept at $-12^{\circ} \mathrm{C}$, overnight. To prepare PVA-based composite cryogels, $125 \mathrm{mg}$ PS-DVB microparticles (dry weight) were washed twice with 5\% (w/v) PVA and once with $4.5 \%(\mathrm{w} / \mathrm{v})$ PVA in $0.1 \mathrm{M} \mathrm{HCl}$, followed by centrifugation at $6,700 \mathrm{~g}$ for $4 \mathrm{~min}$ after each washing step. The microparticles were resuspended in acidic 4.5\% PVA solution to a final volume of $1.2 \mathrm{~mL}$ and the cryogels were produced as described above. After defrosting, the cryogels were washed with distilled water and the residual aldehyde groups were reduced by circulating $0.1 \mathrm{M} \mathrm{NaBH}_{3} \mathrm{CN}$ solution in $0.1 \mathrm{M}$ sodium bicarbonate buffer $(\mathrm{pH} 9.2)$ through the cryogels at a flow rate of $0.5 \mathrm{~mL} / \mathrm{min}$ at $4^{\circ} \mathrm{C}$ for $4 \mathrm{~h}$. Thereafter, the cryogels were extensively washed with distilled water.

To prepare monolithic agarose-based composite cryogels, agarose was dissolved at $3 \%(\mathrm{w} / \mathrm{v})$ in boiling deionized water and the $\mathrm{pH}$ was adjusted to $12.5-13.0$ with $\mathrm{NaOH}$. The agarose solution was added to $125 \mathrm{mg}$ PS-DVB microparticles, mixed and centrifuged at $14,100 \mathrm{~g}$ for $30-60 \mathrm{sec}$. The particles were resuspended in the agarose solution to a final volume of $1.2 \mathrm{~mL}$ and the suspension was transferred into $2 \mathrm{~mL}$ syringes. The cryogels were immediately placed into the liquid bath cryostat $\left(-32^{\circ} \mathrm{C}, 30 \mathrm{~min}\right)$ and thereafter into the air bath 
cryostat $\left(-12^{\circ} \mathrm{C}\right.$, overnight). To prepare agarose cryogels, the above procedure was performed without the addition of microparticles. After defrosting, the cryogels were extensively washed with distilled water and stored in $20 \%(\mathrm{v} / \mathrm{v})$ ethanol at $4^{\circ} \mathrm{C}$.

2.4. Scanning Electron Microscopy. Discs of $4 \mathrm{~mm}$ thickness were cut from the top and bottom of the cryogels and fixed overnight in $2.5 \%(\mathrm{v} / \mathrm{v})$ glutaraldehyde in $0.12 \mathrm{M}$ sodium phosphate buffer ( $\mathrm{pH}$ 7.2). Samples were dehydrated with increasing concentrations of ethanol $(30,50,75$, and $99.5 \%$ $\mathrm{v} / \mathrm{v})$, critical point dried (CPD 030 critical point dryer, BALTEC AG, Balzers, Liechtenstein), and coated with goldpalladium $(40: 60)$ using a Polaron SC7640 High Resolution Sputter Coater (Thermo VG Microtech, West Sussex, Great Britain). The samples were examined on a JEOL JSM-5600 LV scanning electron microscope at an acceleration voltage of $7 \mathrm{kV}$. The pore sizes of the cryogels were estimated based on these images.

2.5. Flow Characteristics of the Cryogels. The flow rate of water through particle-free and composite cryogels was assessed at different pressures. The hydrostatic pressure was adjusted to $1,4,8$, and $15 \mathrm{kPa}$ and the time and volume of water flowing through the monoliths were recorded to calculate the flow rate in $\mathrm{mL} / \mathrm{min}$.

2.6. Particle Leakage. Particle-free cryogels and composite cryogels were washed with 4-5 column volumes of deionized water. Subsequently, $25 \mathrm{~mL}$ of deionized water was passed through plain cryogels and composite cryogels at a flow rate of $1 \mathrm{~mL} / \mathrm{min}$. The quantity of particles released from the cryogels was determined in the flow through by light obscuration using a HIAC Royco device (Pacific Scientific Instruments, Oregon, USA).

2.7. Adsorption of Toxins Related to Liver Failure. Human plasma was spiked with $300 \mu \mathrm{M}$ bilirubin, $100 \mu \mathrm{M}$ cholic acid, $100 \mu \mathrm{M}$ tryptophan, and $2 \mathrm{mM}$ phenol. Adsorption of bilirubin and cholic acid to the cryogels was assessed under static and dynamic conditions. For the static setup, plain cryogels and composite cryogels were cut into $4 \mathrm{~mm}$ slices, which were incubated with $7.65 \mathrm{~mL}$ of spiked plasma at $37^{\circ} \mathrm{C}$ with gentle shaking. For comparison, the equivalent amount of plain PS-DVB microparticles (125 mg) was incubated with spiked plasma. For the dynamic setup, $13 \mathrm{~mL}$ of spiked plasma was recirculated through the plain and composite cryogels at a flow rate of $1 \mathrm{~mL} / \mathrm{min}$. For both setups, samples were taken after $0,15,30,60$, and $120 \mathrm{~min}$. After immediate centrifugation (18,600 g, $3 \mathrm{~min}$ ), the supernatants were stored at $-20^{\circ} \mathrm{C}$ until further analysis. Bilirubin, cholic acid, and albumin were quantified using a Hitachi 902 analyzer (Roche Diagnostics, Mannheim, Germany).

2.8. Adsorption of Cytokines. Recombinant human tumor necrosis factor-alpha (TNF- $\alpha$ ) and interleukin-6 (IL-6) were purchased from R\&D Systems, Minneapolis, USA. Human plasma was spiked with TNF- $\alpha$ and IL- 6 at target concentrations of $500 \mathrm{pg} / \mathrm{mL}$ and $200 \mathrm{pg} / \mathrm{mL}$, respectively. Particle-free cryogels and composite cryogels containing $125 \mathrm{mg}$ PS-DVB microparticles were cut into slices $(4 \mathrm{~mm})$ and incubated at a $10 \%(\mathrm{v} / \mathrm{v})$ microparticle-to-plasma-ratio at $37^{\circ} \mathrm{C}$ with gentle shaking. Samples were taken after 0,15 , and $60 \mathrm{~min}$. For comparison, the equivalent amount of plain PS-DVB microparticles $(125 \mathrm{mg})$ was incubated with spiked plasma as described above. Cytokines were quantified by ELISA (R\&D Systems Quantikine, Minneapolis, USA).

2.9. Activation of Coagulation. The blood compatibility of PS-DVB microparticles, particle-free cryogels, and composite cryogels was assessed using two global coagulation tests, namely, prothrombin time (PT), which detects coagulation deficiencies in the extrinsic coagulation pathway, and activated partial thromboplastin time (aPTT), which detects coagulation deficiencies in the intrinsic coagulation pathway. Single $4 \mathrm{~mm}$ slices of the composite cryogels were incubated with plasma at a $10 \%(\mathrm{v} / \mathrm{v})$ microparticle-to-plasma-ratio for $60 \mathrm{~min}$ at $37^{\circ} \mathrm{C}$. Equivalent volumes of plain PS-DVB particles served as controls. Thereafter, the samples were centrifuged for $3 \mathrm{~min}$ at 18,600 g. PT and aPTT were determined from the supernatants using a Sysmex CA-500 coagulation analyzer (Sysmex Corporation, Kobe, Japan) and the reagents Dade Innovin and Dade Actin FS (both from Siemens Healthcare Diagnostic Products GmbH, Marburg, Germany) according to the instructions of the manufacturers.

\section{Results and Discussion}

3.1. Characterization of Plain Cryogels. Scanning electron microscopy (SEM) revealed that the particle-free monolithic PVA cryogels possessed randomly shaped pores (Figures $1(\mathrm{a})$ and $1(\mathrm{~b}))$. The majority of the pores had a diameter of $100-140 \mu \mathrm{m}$. In addition, smaller pores with a diameter of $10-60 \mu \mathrm{m}$ were observed. Plain PVA cryogels had dense pore walls consisting of thin polymerized layers of PVA (Figure 1(b)). PVA cryogels yielded maximum flow rates of $3.6 \mathrm{~mL} / \mathrm{min}$ at $8 \mathrm{kPa}$. At higher pressures, the flow rates did not increase due to mechanical compression of the gels, which was associated with increasing resistance to the flow (Table 1).

In contrast to PVA-based cryogels, agarose-based cryogels showed regular elongated pores with a length of several hundred micrometers and a width of about $60-70 \mu \mathrm{m}$. The monolithic agarose-based cryogels had very thin pore walls, from which small irregular protrusions were extending (Figure 2(a)). The surface of the pore walls was smooth and non-porous (Figure 2(b)). Agarose cryogels were less permeable than PVA cryogels. The flow rate through agarose cryogels reached a maximum of $2.9 \mathrm{~mL} / \mathrm{min}$ at $4 \mathrm{kPa}$ and decreased at a pressure of $15 \mathrm{kPa}$ (Table 1).

3.2. Composite Cryogels. When developing composite cryogels, we aimed to embed significant amounts of PS-DVB microparticles to achieve high adsorption capacities. Suspending $125 \mathrm{mg}$ PS-DVB microparticles in $1.2 \mathrm{~mL}$ of PVA solution corresponded to an approximately twofold weight 


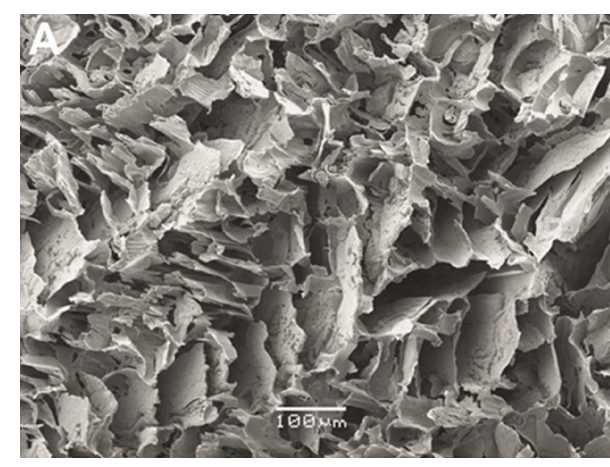

(a)

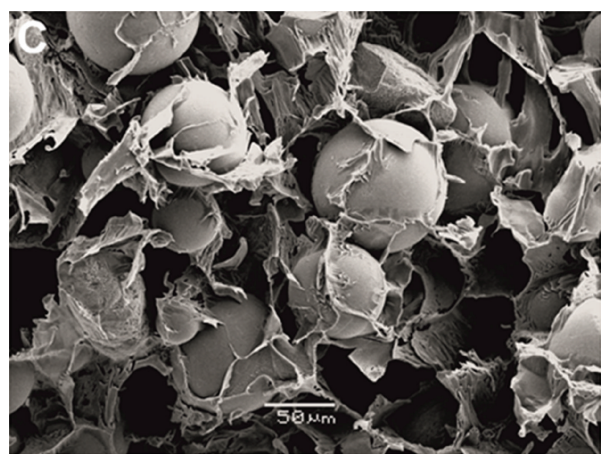

(c)

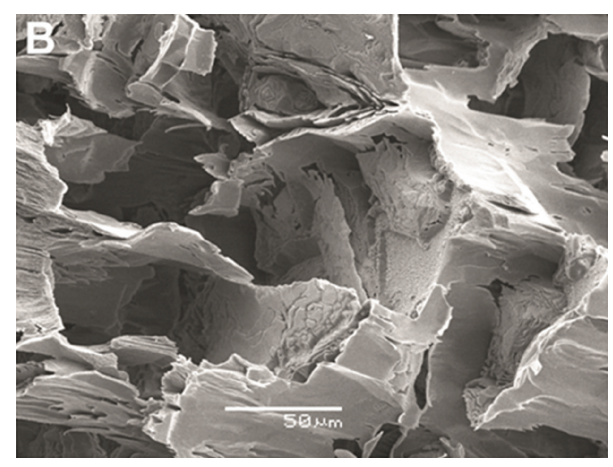

(b)

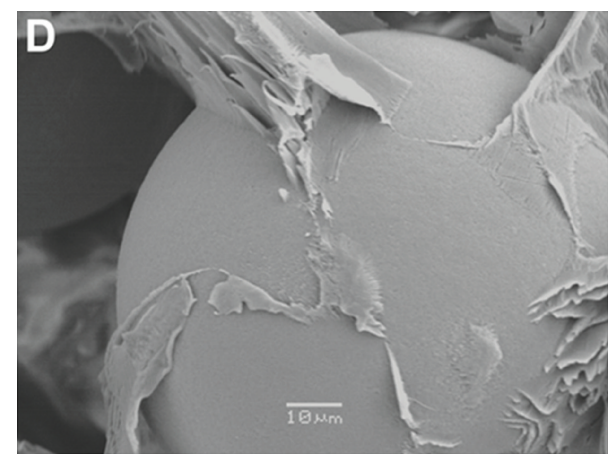

(d)

FIGURE 1: Scanning electron micrographs of plain PVA cryogels (a)-(b) and PVA-based composite cryogels loaded with 125 mg PS-DVB microparticles (c)-(d).

TABLE 1: Flow characteristics of particle-free and composite cryogels $(n \geq 4)$.

\begin{tabular}{|c|c|c|c|c|c|}
\hline \multirow{2}{*}{ Cryogel matrix } & \multirow{2}{*}{ PS-DVB } & \multicolumn{4}{|c|}{ Flow rate $[\mathrm{mL} / \mathrm{min}]$} \\
\hline & & $1 \mathrm{kPa}$ & $4 \mathrm{kPa}$ & $8 \mathrm{kPa}$ & $15 \mathrm{kPa}$ \\
\hline \multirow[t]{2}{*}{ PVA } & - & $1.0 \pm 0.8$ & $3.2 \pm 1.2$ & $3.6 \pm 2.4$ & $3.2 \pm 1.8$ \\
\hline & $125 \mathrm{mg}$ & $0.5 \pm 0.7$ & $1.5 \pm 1.4$ & $2.4 \pm 0.6$ & $4.2 \pm 1.6$ \\
\hline \multirow[t]{2}{*}{ Agarose } & - & $0.8 \pm 0.2$ & $2.9 \pm 1.8$ & $2.8 \pm 1.8$ & $1.8 \pm 1.2$ \\
\hline & $125 \mathrm{mg}$ & $1.2 \pm 0.7$ & $4.0 \pm 3.0$ & $5.9 \pm 4.8$ & $6.7 \pm 6.0$ \\
\hline
\end{tabular}

excess of PS-DVB microparticles over PVA. The pores within the resulting composite cryogels were in the range of $10-60 \mu \mathrm{m}$, and $40-60 \mu \mathrm{m}$ pores were the most common (Figure 1(c)). Thus, the pore size was reduced as compared to particle-free PVA cryogels. Overall, the macroporous structure of plain cryogels significantly differed from that of composite cryogels, as embedded microparticles occupied the cryogel macropores.

The flow rates for PVA-based composite cryogels increased in a linear way with increasing pressure. The flow rate for PVA-based composite cryogels loaded with $125 \mathrm{mg}$ microparticles was $4.2 \mathrm{~mL} / \mathrm{min}$ at $15 \mathrm{kPa}$. For agarose-based composite cryogels the pore sizes varied between 10 and $150 \mu \mathrm{m}$ (Figure 2(c)). Agarose-based composite cryogels showed improved mechanical stability in comparison to particle-free cryogels, as they could be tested at a pressure of $15 \mathrm{kPa}$, yielding flow rates of $6.7 \mathrm{~mL} / \mathrm{min}$ when loaded with $125 \mathrm{mg}$ of microparticles. In conclusion, the mechanical strength of both PVA- and agarose-based cryogels was improved due to the integration of PS-DVB microparticles. Therefore, the composite cryogels allowed the testing at higher pressures and higher flow rates could be achieved as compared to plain cryogels. In the case of PVA composite cryogels, the PS-DVB microparticles were covered by PVA layers and showed a stable integration into the cryogel (Figures 1(c) and 1(d)). In contrast, the PS-DVB particles embedded into agarose-based cryogels appeared to be less tightly incorporated into the gel structure but were rather mechanically entrapped inside the pores (Figure 2(c)).

3.3. Particle Leakage. None of the effluents collected from the composite cryogels contained microparticles in the size range of $40-100 \mu \mathrm{m}$. Thus, the composite cryogels did not need filters to support the embedded microparticles, which were withheld inside the macroporous polymer structure. 


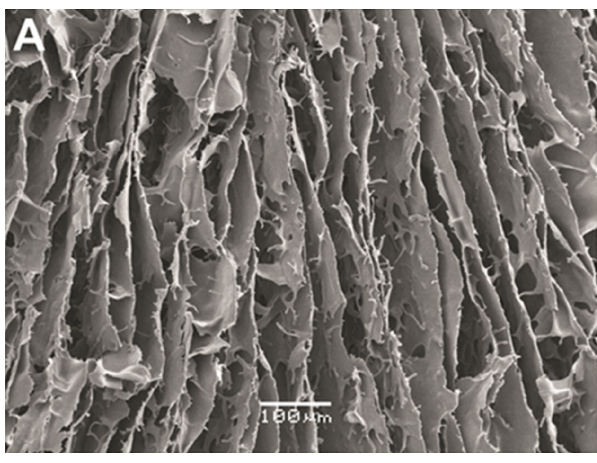

(a)

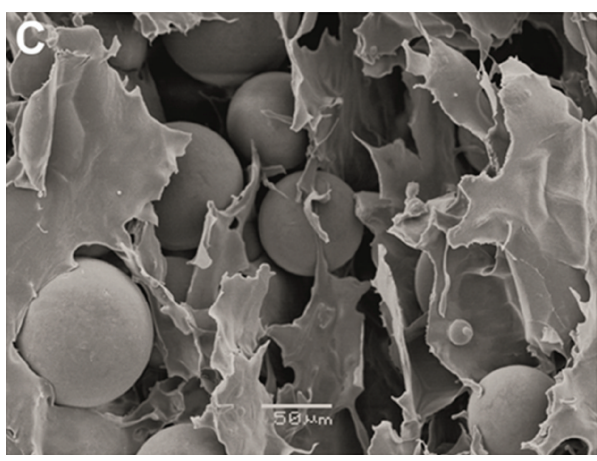

(c)

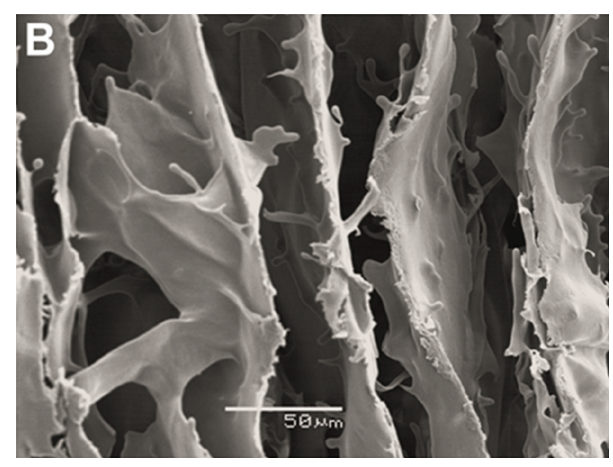

(b)

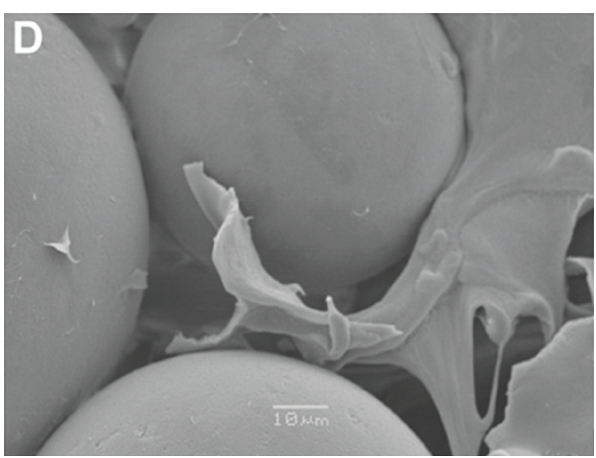

(d)

FIGURE 2: Scanning electron micrographs of plain agarose cryogels (a)-(b) and agarose-based composite cryogels loaded with 125 mg PS-DVB microparticles (c)-(d).

TABLE 2: Adsorption of bilirubin under static and dynamic conditions after 15 and $60 \min (n=3)$.

\begin{tabular}{|c|c|c|c|c|}
\hline & \multicolumn{4}{|c|}{$\mu \mathrm{mol}$ bilirubin adsorbed per g PS-DVB } \\
\hline & \multicolumn{2}{|c|}{ Static } & \multicolumn{2}{|c|}{ Dynamic } \\
\hline & $15 \mathrm{~min}$ & $60 \mathrm{~min}$ & $15 \mathrm{~min}$ & $60 \mathrm{~min}$ \\
\hline PS-DVB & $3.3 \pm 0.2$ & $4.3 \pm 0.2$ & n.a. ${ }^{\mathrm{a}}$ & n.a. ${ }^{\mathrm{a}}$ \\
\hline PVA + 125 mg PS-DVB & $0.5 \pm 0.4$ & $1.3 \pm 0.3$ & $0.6 \pm 0.2$ & $2.1 \pm 0.7$ \\
\hline Agarose +125 mg PS-DVB & $1.2 \pm 0.4$ & $2.8 \pm 0.9$ & $2.6 \pm 1.9$ & $5.0 \pm 0.5$ \\
\hline
\end{tabular}

${ }^{a}$ Not applicable.

3.4. Adsorption of Toxins Related to Liver Failure. The adsorption characteristics of parent PS-DVB microparticles and PS-DVB microparticles embedded into cryogels were investigated under static and dynamic conditions. Under static conditions, the adsorption of bilirubin was reduced by a factor of 3.3 and 1.5, respectively, for PS-DVB microparticles embedded into PVA- and agarose-based cryogels as compared to the parent microparticles (Table 2). Likewise, cholic acid adsorption was reduced by a factor of 4.5 and 3.4, respectively, for PS-DVB microparticles embedded into PVA- and agarose-based cryogels as compared to the parent PS-DVB microparticles (Table 3). Nevertheless, cholic acid was reduced to $10 \%$ and $6.6 \%$ of the initial concentration for PVA-based and agarose-based cryogels containing $125 \mathrm{mg}$ microparticles, respectively.

The importance of convective mass transfer for the adsorption of toxins was reflected by the results of dynamic experiments where the adsorbed amounts of both, bilirubin,
TABle 3: Adsorption of cholic acid under static and dynamic conditions after $60 \mathrm{~min}(n=3)$.

\begin{tabular}{lcc}
\hline & \multicolumn{2}{c}{$\begin{array}{c}\text { Cholic acid adsorbed per } \\
\mu \text { mol/g PS-DVB }\end{array}$} \\
& Static & Dynamic \\
\hline PS-DVB & $22.2 \pm 0.4^{\text {a }}$ & n.a. \\
PVA + 125 mg PS-DVB & $4.9 \pm 0.2$ & $9.6 \pm 0.5$ \\
Agarose + 125 mg PS-DVB & $6.5 \pm 0.3$ & $9.4 \pm 0.5$ \\
\hline
\end{tabular}

${ }^{\mathrm{a}}$ Calculated based on an assay with $1 \%(\mathrm{v} / \mathrm{v})$ adsorbent-to-plasma-ratio.

${ }^{\mathrm{b}}$ Not applicable.

and cholic acid were generally higher than under static conditions (Tables 2 and 3). Agarose-based composite cryogels performed superiorly with respect to bilirubin removal than PVA-based composite cryogels $(5.0 \pm 0.5 \mu \mathrm{mol} / \mathrm{g}$ versus 


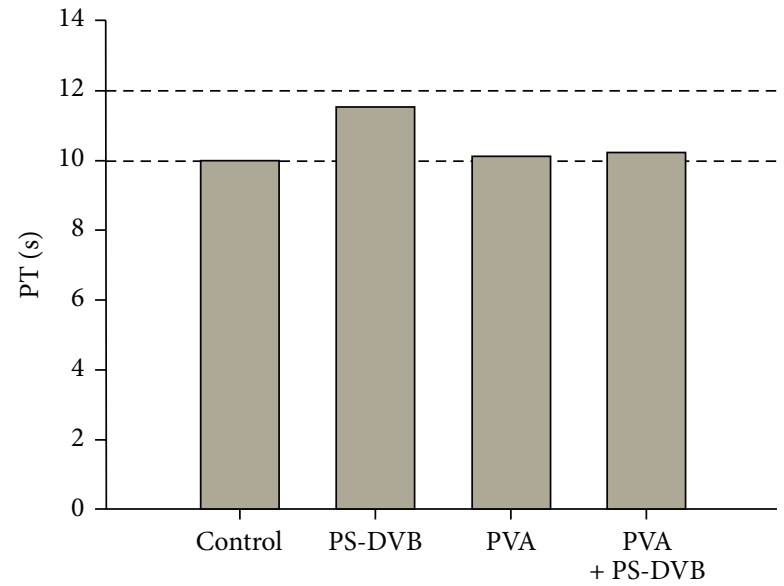

(a)

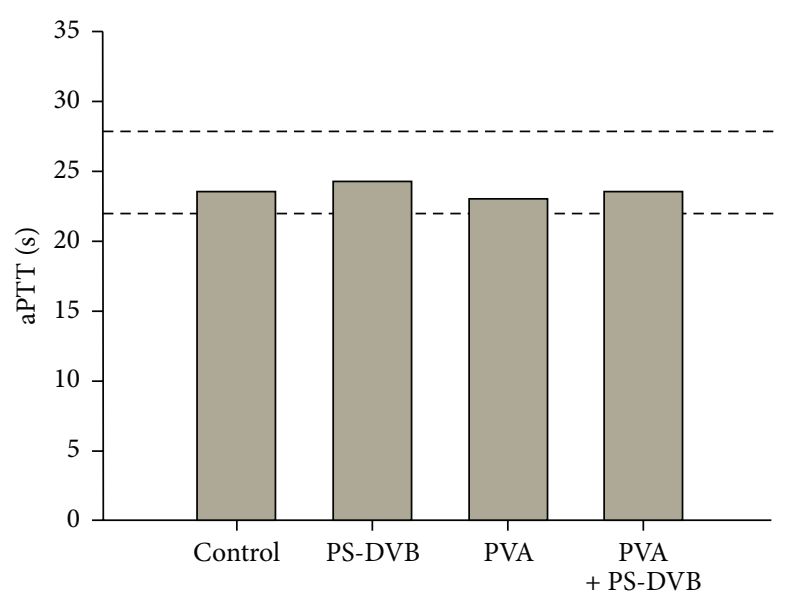

(c)

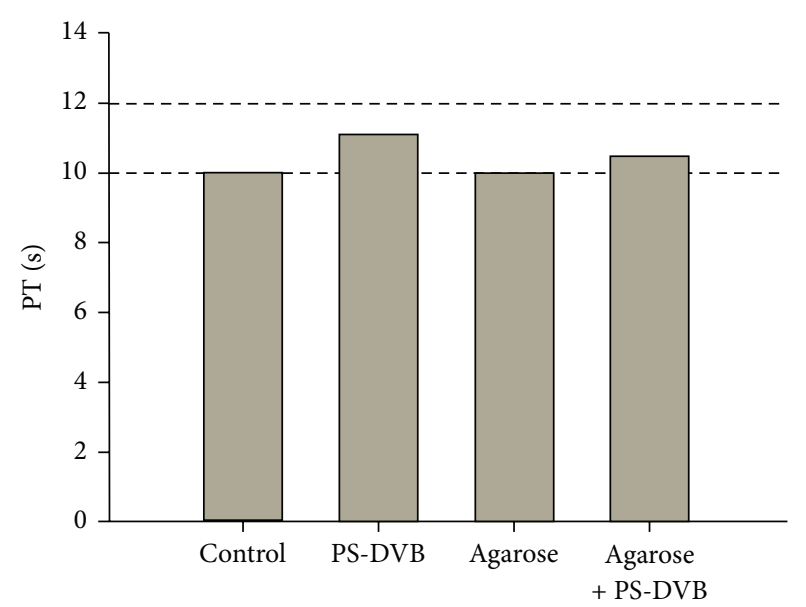

(b)

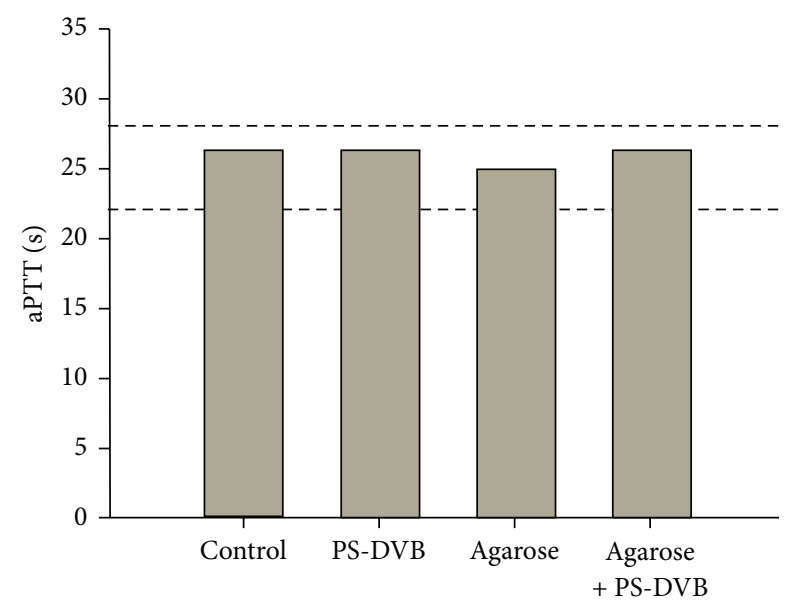

(d)

FIGURE 3: Prothrombin time (PT) and activated partial thromboplastin time (aPTT) for PVA- and agarose-based composite cryogels and parent PS-DVB microparticles. The horizontal lines refer to the reference range of the respective coagulation parameter.

$2.1 \pm 0.7 \mu \mathrm{mol} / \mathrm{g}$, Table 2 ), while no difference was observed with respect to cholic acid removal.

The finding that the accessibility of the pores had a greater influence on the adsorption of bilirubin as compared to cholic acid may be explained by the different affinities of cholic acid and bilirubin for albumin: bilirubin is strongly albuminbound with an association constant of $9.5 \times 10^{7} \mathrm{M}^{-1}$, corresponding to an unbound fraction at the equilibrium of less than $0.1 \%$ [24], while the association constant of cholic acid is $0.3 \times 10^{4} \mathrm{M}^{-1}$, corresponding to a free fraction of cholic acid at the equilibrium of about $16 \%$ [25]. Thus, the diminished accessibility of the pores due to the embedding of PS-DVB microparticles had a stronger influence on the adsorption of bilirubin than on the binding of cholic acid, because the pores remain accessible to the free fraction of the latter metabolite.

The incorporation of $250 \mathrm{mg}$ PS-DVB microparticles into cryogels resulted in a higher decrease in bilirubin and cholic acid concentrations in comparison to cryogels loaded with $125 \mathrm{mg}$ microparticles. However, when calculating the amount of metabolites adsorbed per gram of microparticles, the less densely loaded cryogel composites eliminated bilirubin and cholic acid more efficiently, both under static and dynamic conditions (data not shown).

3.5. Adsorption of Cytokines. Cytokines are mediators of inflammation and their removal from the blood may be relevant both in liver failure and as a supportive treatment option for septic patients $[26,27]$. Human plasma was spiked with TNF- $\alpha$ and IL- 6 to yield concentrations of $535 \mathrm{pg} / \mathrm{mL}$ (TNF- $\alpha$ ) and $182 \mathrm{pg} / \mathrm{mL}$ (IL-6). Cytokine adsorption was compared for the parent microparticles and the composite cryogels containing PS-DVB microparticles (Table 4). Remarkably, TNF- $\alpha$, which is a $51 \mathrm{kD}$ homotrimer in its bioactive form, was removed by plain PS-DVB microparticles to a greater extent than IL-6 $(21 \mathrm{kD})$. This is in contrast to the hypothesis that adsorption would be more efficient for smaller solutes due to better accessibility of the pores. This finding will be further investigated and might eventually be due to deoligomerization of TNF- $\alpha$ into its subunits. 
TABLE 4: Adsorption of TNF- $\alpha$ and IL- 6 on PS-DVB microparticles and composite cryogels after 60 min under static conditions $(n=3)$.

\begin{tabular}{lcc}
\hline & pmol cytokine adsorbed per g PS-DVB \\
& TNF- $\alpha$ & IL-6 \\
\hline PS-DVB & $0.27 \pm 0.01$ & $0.18 \pm 0.01$ \\
PVA + 125 mg PS-DVB & $0.10 \pm 0.03$ & $0.06 \pm 0.04$ \\
Agarose + 125 mg PS-DVB & $0.11 \pm 0.01$ & $0.11 \pm 0.05$ \\
\hline
\end{tabular}

Similar to the case of albumin-bound liver toxins, embedding of the PS-DVB microparticles into the cryogels resulted in decreased cytokine adsorption. This may be explained by the formation of a hydrophilic polymer layer on the PS-DVB microparticles during embedment into the cryogels, which reduces hydrophobic interactions between PS-DVB and the target cytokines.

3.6. Blood Compatibility. Incubation of PS-DVB microparticles with plasma did not result in an increase of prothrombin time and activated partial thromboplastin time. Both PT and aPTT also stayed in the normal range for agarose- and PVAbased composite cryogels, indicating good blood compatibility for PS-DVB microparticles as well as for composite cryogels (Figure 3).

\section{Conclusions}

PS-DVB microparticles with defined porosity are efficient adsorbents for both albumin-bound toxins related to liver failure and cytokines. In this study, PS-DVB microparticles were embedded into monolithic cryogels. The resulting composite cryogels did not require filters to support microparticles and were mechanically stable when perfused with human plasma at flow rates of 4 to $7 \mathrm{~mL} / \mathrm{min}$. The composite cryogels efficiently adsorbed albumin-bound hydrophobic metabolites related to liver failure (bilirubin, cholic acid) and cytokines (TNF- $\alpha$, IL-6). The molecular adsorption characteristics of PS-DVB microparticles were influenced by the embedding procedure, and the accessibility of the pores was diminished due to the formation of a polymer layer on the surface of the particles. This resulted in a decrease of both adsorption capacity and rates of adsorption as compared to the parent microparticles.

The specific adsorption, that is, the amount of target substance bound per $\mathrm{g}$ of adsorbent, was higher for composite cryogels containing $125 \mathrm{mg}$ of particles as compared to the more densely packed composite cryogels containing $250 \mathrm{mg}$ of particles, indicating that there is an optimal density of particles within the composite cryogel. The composite cryogels exhibited good blood compatibility, as global coagulation parameters (prothrombin time and activated partial thromboplastin time) remained stable after incubation of the cryogels with plasma. Thus, we conclude that composite cryogels containing PS-DVB microparticles are suitable as adsorption modules to deplete toxic metabolites from plasma for research and production purposes.

\section{Acknowledgments}

This work was funded by the European commission within the MONACO-EXTRA project "Monolithic Adsorbent Columns for Extracorporeal Medical Devices and Bioseparations" (FP7-PEOPLE-2007-3-1-IAPP). The author thank Ute Fichtinger (Danube University Krems) for technical support and Dr. Rita Wallén (Lund University) for guidance during SEM studies.

\section{References}

[1] A. Jungbauer and R. Hahn, "Monoliths for fast bioseparation and bioconversion and their applications in biotechnology," Journal of Separation Science, vol. 27, no. 10-11, pp. 767-778, 2004.

[2] H. Kirsebom, D. Topgaard, I. Y. Galaev, and B. Mattiasson, "Modulating the porosity of cryogels by influencing the nonfrozen liquid phase through the addition of inert solutes," Langmuir, vol. 26, no. 20, pp. 16129-16133, 2010.

[3] V. I. Lozinsky, I. Y. Galaev, F. M. Plieva, I. N. Savina, H. Jungvid, and B. Mattiasson, "Polymeric cryogels as promising materials of biotechnological interest," Trends in Biotechnology, vol. 21, no. 10, pp. 445-451, 2003.

[4] F. M. Plieva, I. Y. Galaev, W. Noppe, and B. Mattiasson, "Cryogel applications in microbiology," Trends in Microbiology, vol. 16, no. 11, pp. 543-551, 2008.

[5] M. B. Dainiak, I. U. Allan, I. N. Savina et al., "Gelatin-fibrinogen cryogel dermal matrices for wound repair: preparation, optimisation and in vitro study," Biomaterials, vol. 31, no. 1, pp. 67-76, 2010.

[6] H. Kirsebom, M. R. Aguilar, J. San Roman, M. Fernandez, M. A. Prieto, and B. Bondar, "Macroporous scaffolds based on chitosan and bioactive molecules," Journal of Bioactive and Compatible Polymers, vol. 22, no. 6, pp. 621-636, 2007.

[7] C. T. Tsao, C. H. Chang, Y. D. Li et al., "Development of chitosan/dicarboxylic acid hydrogels as wound dressing materials," Journal of Bioactive and Compatible Polymers, vol. 26, no. 5, pp. 519-536, 2011.

[8] K. Bloch, V. I. Lozinsky, I. Y. Galaev et al., "Functional activity of insulinoma cells (INS-1E) and pancreatic islets cultured in agarose cryogel sponges," Journal of Biomedical Materials Research A, vol. 75, no. 4, pp. 802-809, 2005.

[9] A. Jain, Y. T. Kim, R. J. McKeon, and R. V. Bellamkonda, "In situ gelling hydrogels for conformal repair of spinal cord defects, and local delivery of BDNF after spinal cord injury," Biomaterials, vol. 27, no. 3, pp. 497-504, 2006.

[10] M. E. Mawad, S. Cekirge, E. Ciceri, and I. Saatci, "Endovascular treatment of giant and large intracranial aneurysms by using a combination of stent placement and liquid polymer injection," Journal of Neurosurgery, vol. 96, no. 3, pp. 474-482, 2002.

[11] S. Sakai, K. Kawabata, T. Ono, H. Ijima, and K. Kawakami, "Development of mammalian cell-enclosing subsieve-size agarose capsules $(<100 \mu \mathrm{m})$ for cell therapy," Biomaterials, vol. 26, no. 23, pp. 4786-4792, 2005.

[12] C. Weis, E. K. Odermatt, J. Kressler, Z. Funke, T. Wehner, and D. Freytag, "Poly(vinyl alcohol) membranes for adhesion prevention," Journal of Biomedical Materials Research B: Applied Biomaterials, vol. 70, no. 2, pp. 191-202, 2004.

[13] S. Gupta, T. J. Webster, and A. Sinha, "Evolution of PVA gels prepared without crosslinking agents as a cell adhesive surface," 
Journal of Materials Science: Materials in Medicine, vol. 22, no. 7, pp. 1763-1772, 2011.

[14] A. Hanora, F. M. Plieva, M. Hedström, I. Y. Galaev, and B. Mattiasson, "Capture of bacterial endotoxins using a supermacroporous monolithic matrix with immobilized polyethyleneimine, lysozyme or polymyxin B," Journal of Biotechnology, vol. 118, no. 4, pp. 421-433, 2005.

[15] I. Perçin, G. Baydemir, B. Ergün, and A. Denizli, "Macroporous PHEMA-based cryogel discs for bilirubin removal," Artificial Cells, Nanomedicine, and Biotechnology, 2012.

[16] R. D. Hughes, "Review of methods to remove protein-bound substances in liver failure," The International Journal of Artificial Organs, vol. 25, no. 10, pp. 911-917, 2002.

[17] W. Laleman, A. Wilmer, P. Evenepoel, C. Verslype, J. Fevery, and F. Nevens, "Review article: non-biological liver support in liver failure," Alimentary Pharmacology and Therapeutics, vol. 23, no. 3, pp. 351-363, 2006.

[18] D. Falkenhagen, W. Strobl, G. Vogt et al., "Fractionated plasma separation and adsorption system: a novel system for blood purification to remove albumin bound substances," Artificial Organs, vol. 23, no. 1, pp. 81-86, 1999.

[19] P. Krisper and R. E. Stauber, “Technology insight: artificial extracorporeal liver support-how does Prometheus compare with MARS?" Nature Clinical Practice Nephrology, vol. 3, no. 5, pp. 267-276, 2007.

[20] D. Falkenhagen, M. Brandl, J. Hartmann, K. H. Kellner, T. Posnicek, and V. Weber, "Fluidized bed adsorbent systems for extracorporeal liver support," Therapeutic Apheresis and Dialysis, vol. 10, no. 2, pp. 154-159, 2006.

[21] V. Weber, I. Linsberger, M. Hauner, A. Leistnr, A. Leistner, and D. Falkenhagen, "Neutral styrene divinylbenzene copolymers for adsorption of toxins in liver failure," Biomacromolecules, vol. 9, no. 4, pp. 1322-1328, 2008.

[22] R. Saich, C. Selden, M. Rees, and H. Hodgson, "Characterization of pro-apoptotic effect of liver failure plasma on primary human hepatocytes and its modulation by molecular adsorbent recirculation system therapy," Artificial Organs, vol. 31, no. 9, pp. 732-742, 2007.

[23] A. Leistner and A. Leistner, "Adsorbing material for blood and plasma cleaning method and for albumin purification," European Patent EP1578526, 2005.

[24] R. Broderson, "Physical chemistry of bilirubin: binding to macromolecules and membranes," in Bilirubin, K. P. M. Heirwegh and S. B. Brown, Eds., vol. 1, pp. 75-123, CRC Press, Boca Raton, Fla, USA, 1982.

[25] A. Roda, G. Cappelleri, R. Aldini, E. Roda, and L. Barbara, "Quantitative aspects of the interaction of bile acids with human serum albumin," Journal of Lipid Research, vol. 23, no. 3, pp. 490-495, 1982.

[26] J. A. Kellum, M. Song, and R. Venkataraman, "Hemoadsorption removes tumor necrosis factor, interleukin-6, and interleukin-10, reduces nuclear factor- $\kappa \mathrm{B}$ DNA binding, and improves short-term survival in lethal endotoxemia," Critical Care Medicine, vol. 32, no. 3, pp. 801-805, 2004.

[27] V. Cantaluppi, V. Weber, C. Lauritano et al., "Protective effect of resin adsorption on septic plasma-induced tubular injury," Critical Care, vol. 14, no. 1, article R4, 2010. 

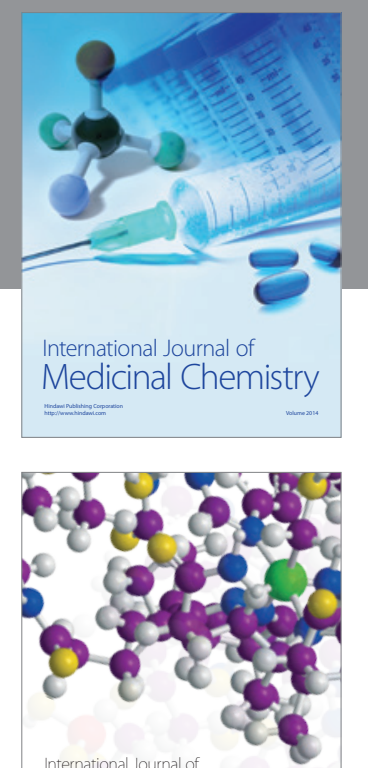

\section{Carbohydrate} Chemistry

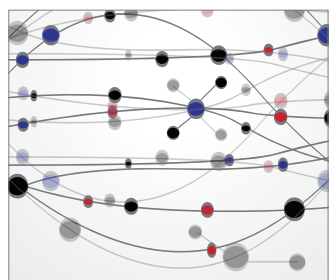

The Scientific World Journal
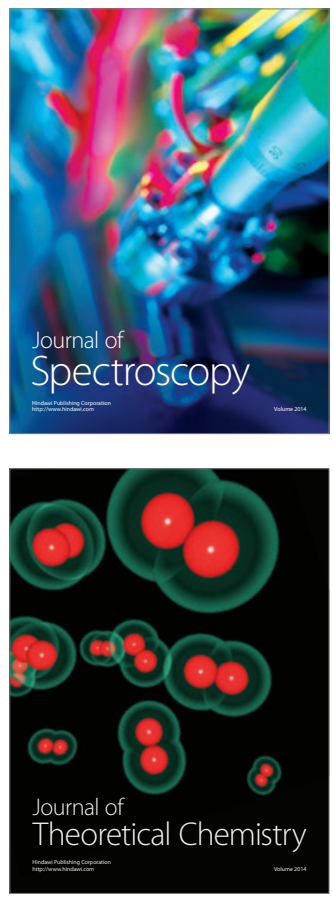
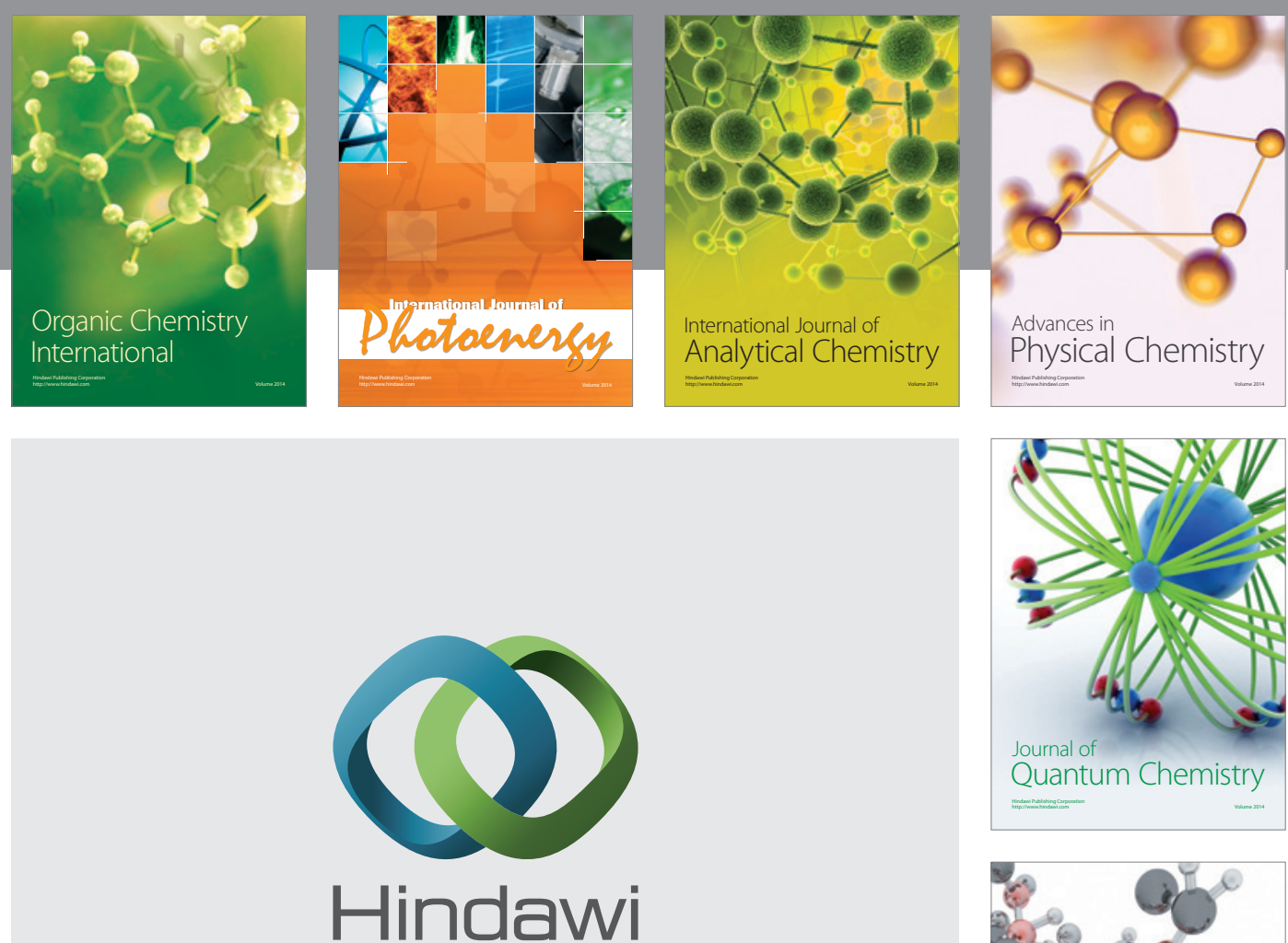

Submit your manuscripts at

http://www.hindawi.com

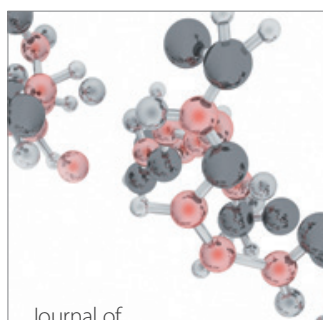

Analytical Methods

in Chemistry

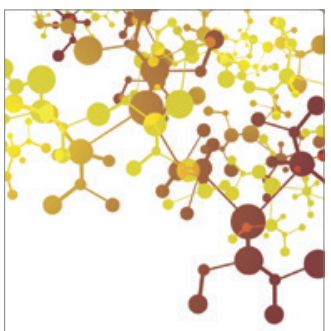

Journal of

Applied Chemistry

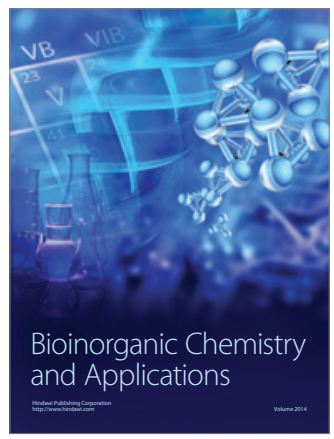

Inorganic Chemistry
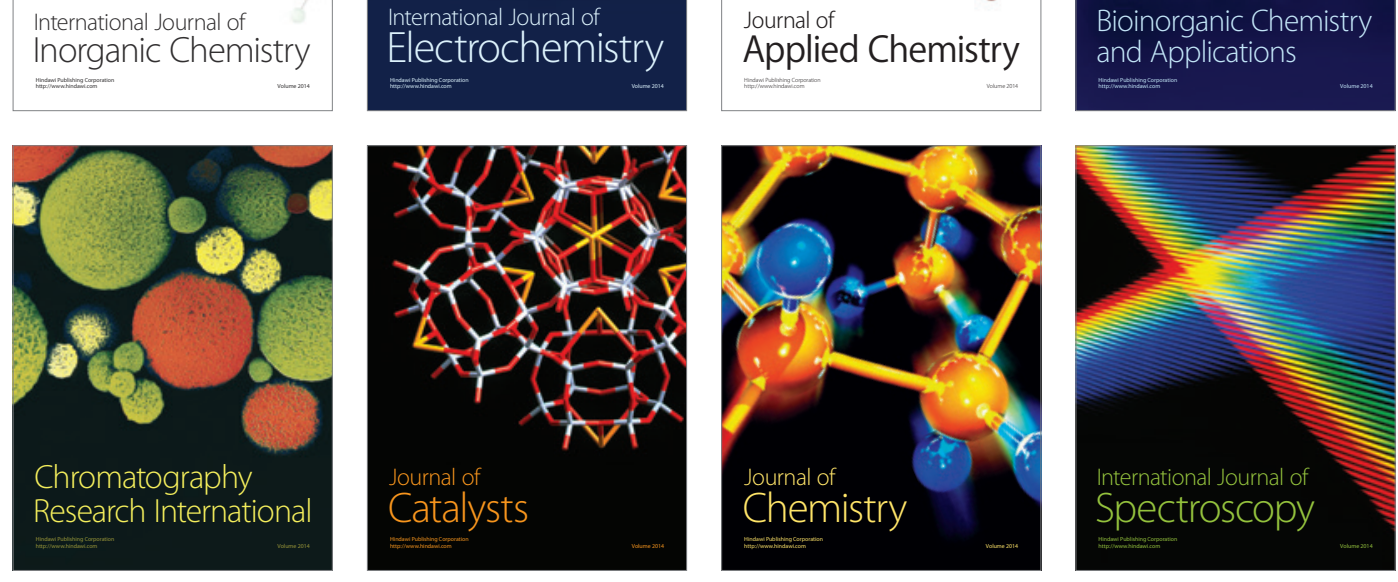